\title{
Feasibility and electromagnetic compatibility study of the ClearPEM front-end electronics for simultaneous PET-MR imaging
}

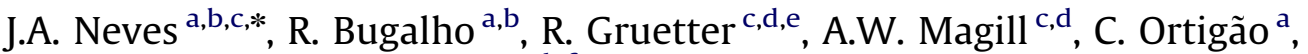 \\ J.C. Silva ${ }^{a}$, R. Silva ${ }^{\mathrm{a}}$, J. Varela ${ }^{\mathrm{a}, \mathrm{b}, \mathrm{f}}$ \\ a LIP - Laboratory of Instrumentation and Experimental Particle Physics, Lisbon, Portugal \\ ${ }^{\mathrm{b}}$ IST-UTL - Instituto Superior Técnico, Technical University of Lisbon, Lisbon, Portugal \\ ${ }^{c}$ Laboratory of Functional and Metabolic Imaging, École Polytechnique Fédérale de Lausanne, Lausanne, Switzerland \\ ${ }^{\mathrm{d}}$ Department of Radiology, University of Lausanne, Lausanne, Switzerland \\ e Department of Radiology, University of Geneva, Geneva, Switzerland \\ ${ }^{\mathrm{f}}$ CERN - European Organization for Nuclear Research, Geneva, Switzerland
}

\section{A R T I C L E I N F O}

Available online 23 August 2012

\section{Keywords:}

PET

ClearPEM

7 Tesla MR

Simultaneous PET-MRI

EMI and EMC

\begin{abstract}
A B S T R A C T
In this work we present a first feasibility study of the ClearPEM technology for simultaneous PET-MR imaging. The mutual electromagnetic interference (EMI) effects between both systems were evaluated on a $7 \mathrm{~T}$ magnet by characterizing the response behavior of the ClearPEM detectors and front-end electronics to pulsed RF power and switched magnetic field gradients; and by analyzing the MR system performance degradation from noise pickup into the RF receiver chain, and from magnetic susceptibility artifacts caused by PET front-end materials.
\end{abstract}

(c) 2012 Elsevier B.V. All rights reserved.

\section{Introduction}

Over the last few years, there have been a growing interest on the development of MR-compatible PET detectors for simultaneous PET-MR imaging. Despite the drawbacks of the APD-based PET detectors, they have proven to be a solid technology over the last decade, being therefore suitable for the development of new PET-MR systems.

The ClearPEM is a dedicated APD-based PET detector for highresolution breast cancer imaging [1]. The basic detector supermodule is composed of 12 modules of $4 \times 8$ LYSO:Ce crystal matrices optically coupled to Hamamatsu S8550 APD arrays on both ends, that are assembled between two front-end electronic boards (FEB). Each FEB integrates two low-noise front-end ASICs (AMS $350 \mathrm{~nm} \mathrm{CMOS,} 70 \mathrm{~mm}^{2}$ ) for the readout (pulse amplification and shaping) of 192 APD input channels; two free-sampling dual 10-bit ADC (running at the system clock, $50 \mathrm{MHz}$ ) for the analog-to-digital data conversion; and a low-voltage differential signaling (LVDS) channel link transmitter (2.4 Gbps) for digital data serialization and transmission to the off-detector data acquisition system [2].

The strong potential of the ClearPEM technology to be adapted to different detector system designs has opened a window of

\footnotetext{
* Corresponding author at: LIP - Laboratory of Instrumentation and Experimental Particle Physics, Lisbon, Portugal. Tel.: +351960 039281.

E-mail address: janeves@lip.pt (J.A. Neves).
}

opportunity on exploiting the feasibility of integration of the technology with MR systems. In this sense, this work intends to present a first study of the electromagnetic compatibility of the ClearPEM detectors and front-end electronics for a combined PETMR imaging system.

\section{Materials and methods}

The mutual electromagnetic interference tests between ClearPEM front-end electronics and the MR system components were carried out at the $7 \mathrm{~T}$ magnet facility at EPFL, Switzerland. The system integrates an actively shielded $7 \mathrm{~T}$ magnet $-68 \mathrm{~cm}$ bore diameter (Magnex Scientific, Oxford, U.K.) with a dedicated head gradient coil (36 cm inner diameter), allowing for maximum gradient strengths of $80 \mathrm{mT} / \mathrm{m}$ switched at slew rates of up to $700 \mathrm{mT} / \mathrm{m} / \mathrm{ms}$ [3].

Proper shielded cables were used for the electrical connections (LV and HV power, clock, control and data links) between the ondetector front-end electronics (placed inside the $7 \mathrm{~T}$ magnet bore) and the power supply units, the off-detector data acquisition system and the measurement equipments placed outside the magnet room. The detector electronics was cooled through a plastic pipe connected to an air cooling device placed outside the magnet room, and the temperature was monitored by an opticalfiber based probe.

The methods described below summarize the experimental tests that were performed to evaluate the mutual electromagnetic 
interference mechanisms between both systems. In the different methods no EMI shielding was used intending to assess the mutual EMI effects in a worst-case scenario.

1. The EMI from the RF coils was assessed as the tolerance of the PET front-end electronics to pulsed RF power. Experimentally, we have used an RF surface coil driven by different RF powers for a pulse sequence corresponding to an RF gate of $2 \mathrm{~ms}$ and with a repetition time of $1 \mathrm{~s}$. A water-filled phantom was used to load the RF coil, maximizing the electromagnetic field coupling (RF power transfer) between the coil and the PET front-end electronics.

2. The EMI from PET front-end electronics was evaluated by measuring the pickup noise to the RF coil with a spectrum analyzer (Agilent 4396B). In a second approach, and intending to assess the effects of picked up noise on the final MR image, an MR scan from a phantom was performed with the surface RF coil in the vicinity of the PET front-end.

3. The effects of the switched magnetic field gradients on the PET front-end electronics were studied with a conventional echoplanar imaging (EPI) readout sequence. The front-end boards were placed perpendicular to the gradient field lines to maximize the area of field penetration through the boards (worst case analysis).

4. A preliminary evaluation of the susceptibility artifacts caused by PET front-end materials was performed by measuring the static $B_{0}$ field distortions via a $B_{0}$ phase-difference mapping acquired with a the ClearPEM supermodule placed in the vicinity of a oil-filled spherical phantom (24 cm diameter).

\section{Experimental results and discussion}

\subsection{EMI from RF coils}

Experimental tests have shown that for an RF power below $50 \mathrm{~W}$, the LVDS analog output of the front-end ASIC rejects common-mode noise pickup from RF coil. However, by increasing the RF power up to $2.8 \mathrm{~kW}$ we have observed a charge saturation of the front-end ASIC dynamic range during the RF pulse; and a self-triggering state induced by RF eddy-currents. This state is characterized by a burst of spurious events remaining up to $1.2 \mathrm{~ms}$ after an RF pulse. Fig. 1 intends to illustrate this effect: the labels (A) corresponds to the ${ }^{176} \mathrm{Lu}$ events from the intrinsic radioactivity of LYSO crystals; (B) represents an RF gate of $2 \mathrm{~ms}$ when the ASIC reaches the saturation; and $(C)$ represents the burst of spurious events after the RF gate.

\subsection{EMI from PET front-end electronics}

Spectrum analyzer measurements of the pickup noise on the RF coils have shown a significant EMI from high-frequency fields radiated from the digital blocks of the front-end electronics, and as demonstrated by Fig. 2, the high-frequency harmonics from the $50 \mathrm{MHz}$ system clock are easily picked up by the RF coils. However, from the acquired spectrum, the 6th harmonic of the clock $(300 \mathrm{MHz}$ ) was found to be the only frequency component entering in the narrow dynamic range of the RF preamplifiers, being responsible for noise propagation into the $\mathrm{RF}$ receiver chain and for the whole MR system SNR degradation.

The effects on the final MR acquired image caused by the SNR degradation of the system are depicted in Fig. 3: the figure on the left shows an acquired MR image of a water-filled phantom with the front-end electronics powered on but with the digital blocks driven by the $50 \mathrm{MHz}$ system clock turned off; in contrast, the figure on the right demonstrates the degradation on the imaging quality when the system clock is turned on (one can clearly see a substantial increase in the image noise, and a set of vertical bands corresponding to imaging artifacts caused by EMI).

Despite the results presented above have been achieved without any EMI shielding, a considerable reduction of the EMI from the PET front-end electronics is expected by introducing a shift on the clock frequency from 50 to $52.5 \mathrm{MHz}$. In this way the 6th harmonic of the clock is kept away from a resonance frequency of $297 \mathrm{MHz}$.

\subsection{EMI effects of switched gradients on PET front-end electronics}

The switching of the magnetic field gradients has shown to produce a non-significant degradation on the PET front-end readout performance. However, in a worst-case scenario with front-end perpendicular to the gradient field lines, high slew-rate gradient transitions $(d B / d x / d t)$ were found to be responsible for induced eddy-currents on front-end boards power and ground planes. From Fig. 4, such eddy-currents are evidenced through induced fluctuations on the ASIC baseline, a ringing distortion

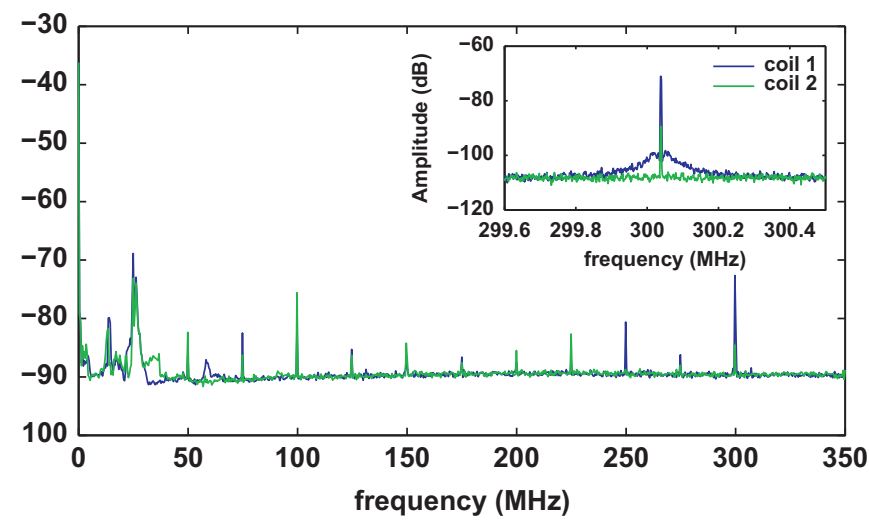

Fig. 2. Pickup noise spectrum from the RF coil system (two circular coils assembled in quadrature). The picture inside corresponds to a narrow band for $300 \mathrm{MHz}$.

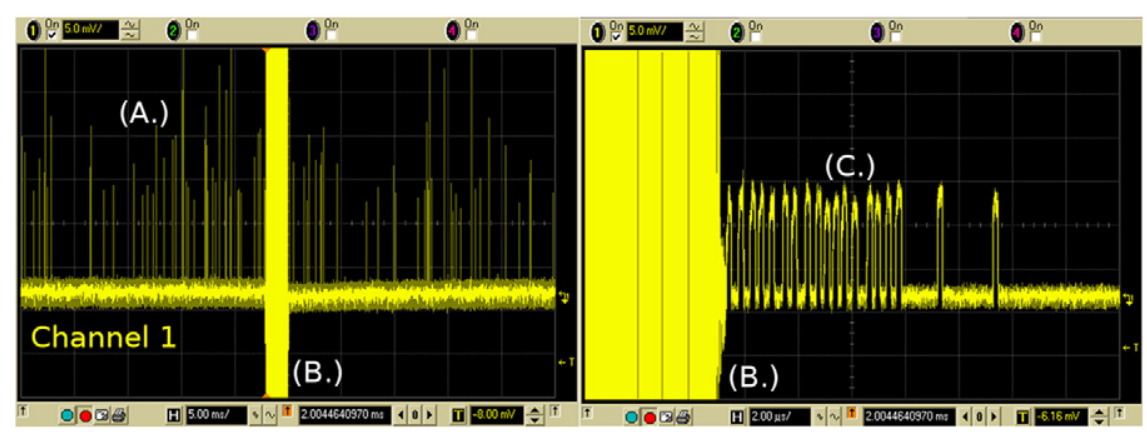

Fig. 1. Analog output of the ClearPEM front-end ASIC seen through an oscilloscope-EMI from pulsed RF power. 

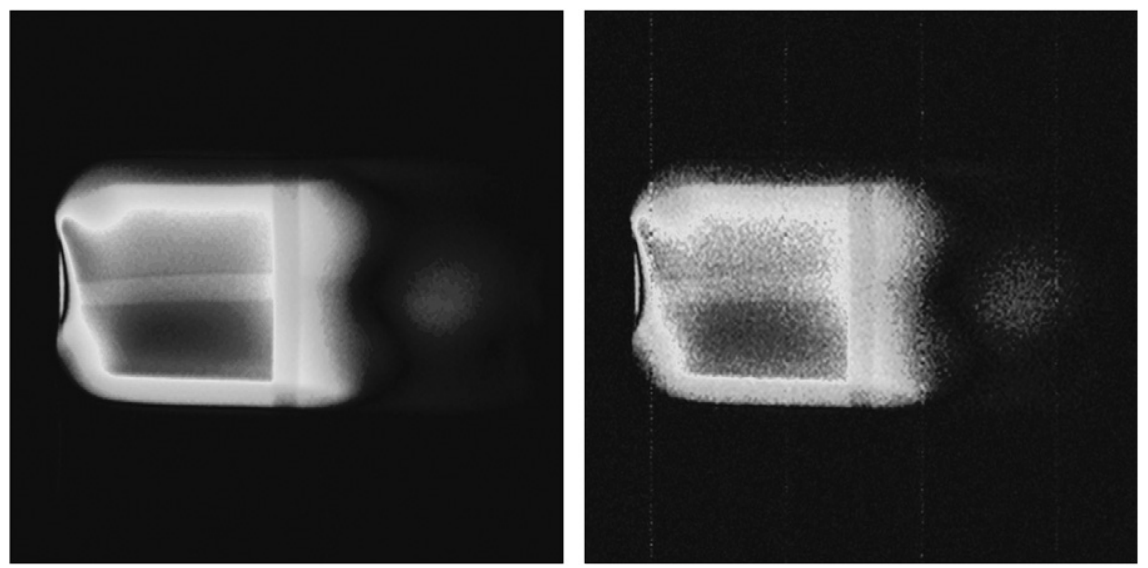

Fig. 3. Effects of noise pickup from the PET front-end electronics on an acquired MR image of a water-filled phantom.

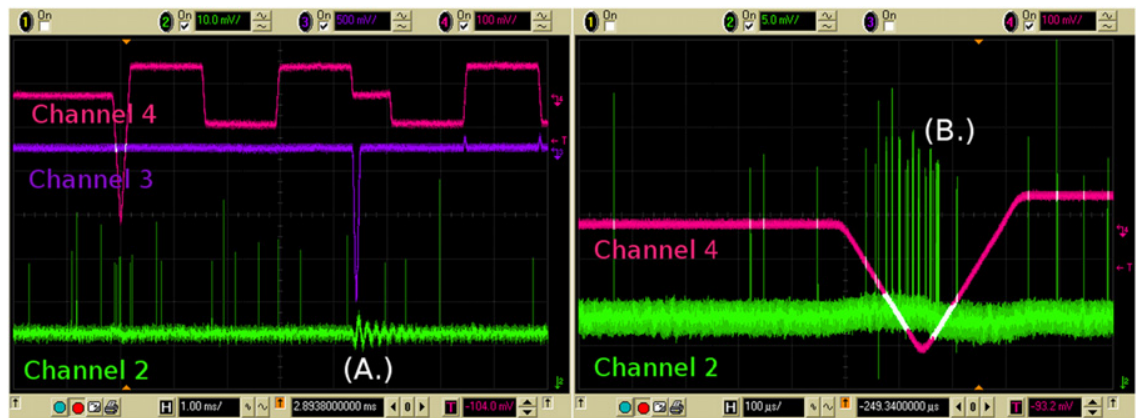

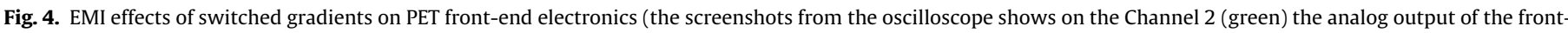

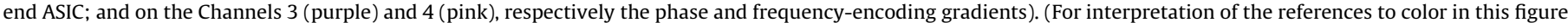
caption, the reader is referred to the web version of this article.)

with an exponential decay time of $600 \mu \mathrm{s}(\mathrm{A})$; and through the generation of bursts of a few tens of spurious events on the analog output of the ASIC (B).

\subsection{Susceptibility artifacts caused by PET front-end materials}

The homogeneity level of the static $B_{0}$ field was evaluated via a phase-difference $B_{0}$ mapping technique. $B_{0}$ field distortions below $10 \mathrm{ppm}$ were observed over an extension of $5 \mathrm{~cm}$ over the DSV of an oil-filled spherical phantom placed in the vicinity of a ClearPEM supermodule inside a head birdcage RF coil. Nickel plated sockets for the assembling of the APD to the front-end boards were found to be responsible for the observed $B_{0}$ field lines distortions.

In conclusion, in this conference we have presented the first results of the feasibility and electromagnetic compatibility study of the ClearPEM detectors and front-end electronics for combined PET-MR imaging. The experimental tests were conducted without any EMI shielding intending to identify the mutual interference effects in a worst case scenario. The main presented results show that the front-end electronics withstands to pulsed RF power and to the strong magnetic field gradients, being feasible for the integration with a $7 \mathrm{~T}$ MR system.

\section{Acknowledgments}

The author would also like to thank colleagues from LIP and CIBM for their contribution, in particular to A.W. Magill. The work of Jorge A. Neves was supported by FCT, Portuguese Foundation for Science and Technology, under the Grant SFRH/BD/33667/ 2009 and by Centre d'Imagerie BioMédicale (CIBM) of the UNIL, UNIGE, HUG, CHUV, EPFL and the Leenaards and Jeantet Foundations.

\section{References}

[1] J.A. Neves, Nuclear Instruments and Methods in Physics Research Section A 628 (2011) 444.

[2] E. Albuquerque, et al., Nuclear Instruments and Methods in Physics Research Section A 598 (2009) 802.

[3] A. vom Endt, J. Riegler, E. Eberlein, F. Schmitt, U. Dorbert, G. Krüger, R. Gruetter, Proceedings of the International Society for Magnetic Resonance in Medicine 15 (2007) 451. 